\title{
Evaluation of the pharmacological activities of RAD1901, a selective estrogen receptor degrader
}

\author{
Suzanne E Wardell, Erik R Nelson*, Christina A Chao, Holly M Alley and \\ Donald P McDonnell
}

Department of Pharmacology and Cancer Biology, Duke University School of Medicine, Box 3813, Durham, North Carolina 27710, USA

*E R Nelson is now at Molecular and Integrative Physiology, University of Illinois at Urbana-Champaign, 523 Burrill Hall, 407 South Goodwin Avenue, Urbana, Illinois 61801, USA
Correspondence should be addressed to D P McDonnell Email

donald.mcdonnell@duke.edu

\begin{abstract}
Endocrine therapy, using tamoxifen or an aromatase inhibitor, remains a first-line treatment for estrogen receptor 1 (ESR1) positive breast cancer. However, tumor resistance limits the duration of response. The clinical efficacy of fulvestrant, a selective ER degrader (SERD) that triggers receptor degradation, has confirmed that ESR1 often remains engaged in endocrine therapy resistant cancers. Recently developed, selective ER modulators (SERMs)/SERD hybrids (SSHs) that facilitate ESR1 degradation in breast cancer cells and reproductive tissues have been advanced as an alternative treatment for advanced breast cancer, particularly in the metastatic setting. RAD1901 is one SSH currently being evaluated clinically that is unique among ESR 1 modulators in that it readily enters the brain, a common site of breast cancer metastasis. In this study, RAD1901 inhibited estrogen activation of ESR1 in vitro and in vivo, inhibited estrogen-dependent breast cancer cell proliferation and xenograft tumor growth, and mediated dose-dependent downregulation of ESR1 protein. However, doses of RAD1901 insufficient to induce ESR1 degradation were shown to result in the activation of ESR 1 target genes and in the stimulation of xenograft tumor growth. RAD1901 is an SSH that exhibits complex pharmacology in breast cancer models, having dose-dependent agonist/ antagonist activity displayed in a tissue-selective manner. It remains unclear how this unique pharmacology will impact the utility of RAD1901 for breast cancer treatment. However, being the only SERD currently known to access the brain, RAD1901 merits evaluation as a targeted therapy for the treatment of breast cancer brain metastases.
\end{abstract}

\section{Key Words}

- selective estrogen receptor degrader

- SERM

- endocrine-resistant breast cancer

- RAD1901

\section{Introduction}

Breast cancer remains the most commonly diagnosed cancer among women and a leading cause of cancer mortality (Carter 2014). While targeted therapies such as the selective estrogen receptor modulator (SERM) tamoxifen and aromatase inhibitors (AIs) are initially effective in the treatment of estrogen receptor alpha 1 (ESR1) positive tumors, de novo and acquired resistance remain an impediment to durable clinical responses, particularly in the setting of advanced disease. However, ESR1 remains a therapeutic target in breast cancers that are resistant to both first- and second-line endocrine interventions (Perey et al. 2007, Riggins et al. 2007), a finding that has prompted the development of i) SERMs with a mechanism distinct from tamoxifen and ii) selective ER degraders

Published by Bioscientifica Ltd 
(SERDs), competitive antagonists whose interaction with ESR1 induce its proteasome-dependent degradation. Fulvestrant, currently the only SERD approved for the treatment of metastatic breast cancer, has been effective as both a first- and second-line therapy in advanced breast cancer (Robertson et al. 2001, Chia et al. 2008, Leo et al. 2009, Robertson et al. 2014); however, the pharmaceutical properties of this drug may prove dose-limiting in relapsed/resistant breast tumors bearing ESR1 mutations known to decrease SERD potency (Robinson et al. 2013, Toy et al. 2013, Jeselsohn et al. 2014). SERDs with improved bioavailability are currently being evaluated in the clinic for efficacy in treating breast cancer patients who have progressed on endocrine therapies (Mayer et al. 2013).

In recent years there has been a high level of interest in exploiting the complexities of ESR1 signaling to identify novel SERMs, compounds whose relative agonist/antagonist activity is manifest in a cell/tissue restricted manner. Motivated by the observation that tamoxifen could exhibit agonist activities in the bone and the endometrium while functioning as an antagonist in breast, investigators have identified and developed a series of ESR1 ligands that display more clinically useful selectivity (i.e., raloxifene (Ralox), ospemifene, and lasofoxifene; Dallenbach-Hellweg et al. 2000, Lindahl et al. 2008, Komm \& Chines 2012). Unexpectedly, these discovery efforts also led to the identification of a series of compounds that exhibit some of the properties of both SERMs and SERDs. These SERM/SERD hybrids (SSHs) have been shown to function as agonists in bone but remarkably inhibit ESR1-action in the reproductive system and in animal models of breast cancer by inducing receptor degradation. The first drug of this class, GW5638/DPC974, was shown to function as a competitive antagonist of ESR1 that induced a conformational change in the receptor that resulted in its being targeted for proteasomal degradation in breast cancer cells (Willson et al. 1997). Importantly, this drug exhibited favorable pharmaceutical properties, inhibited the growth of tamoxifen-resistant breast tumor xenografts and demonstrated efficacy in a small study of patients with advanced, heavily pretreated, breast cancer (Bentrem et al. 2001, Connor et al. 2001, Dardes et al. 2002). Whereas this drug was abandoned for nonscientific reasons, its demonstrated efficacy led others to search for similar molecules that exhibited SSH activity. Of note is i) the identification of ARN810 (GDC-0810; Lai et al. 2015), a structural analogue of GW5638 and ii) the observation that bazedoxifene (BZA), a drug approved for the treatment and prevention of osteoporosis in postmenopausal women, exhibits tissue-selective SERD activity (S Wardell, unpublished observations). Both drugs effectively inhibit the growth of both treatment-naive and tamoxifen-resistant xenograft tumors in mice and are at different stages of clinical development for metastatic breast cancer (Lewis-Wambi et al. 2011, Mayer et al. 2013, Wardell et al. 2013, 2015).

Despite their efficacy in the treatment of postmenopausal osteoporosis, the currently available SERMs and SSHs do not treat the vasomotor instability (hot flushes) associated with menopause. One impediment to the identification of a SERM/SSH modulator for the treatment of hot flashes is the inability to identify compounds that effectively cross the blood-brain barrier. The identification of RAD1901, a SERM that readily enters the brain, was therefore of interest. Preclinical studies showed that RAD1901 mitigated vasomotor symptoms in animal models while also preventing ovariectomy-associated bone loss (Hattersley et al. 2007). Remarkably, however, when the utility of RAD1901 to reduce vasomotor symptoms was evaluated clinically, an unexpectedly complex dose response was observed. Specifically, vasomotor symptoms were improved at the lowest dose evaluated, whereas higher dose administration was found to be ineffective or even to exacerbate symptoms when compared to placebo (O'Dea et al. 2010). Thus, RAD1901 is unique among SERMs in that it displays a complex dose-related agonist/antagonist activity. Although a phase I clinical trial was recently initiated evaluating RAD1901 as a potential therapy for advanced breast cancer (clinical trial \#NCT02338349), it is important to elucidate the mechanism underlying the complex pharmacology of this drug to ensure that it is evaluated in a manner that maximizes its potential for success. Thus, the objective of this study was to probe the molecular mechanism(s) underlying the complex pharmacology of RAD1901 - studies that we believe will inform its optimal clinical development.

\section{Materials and methods}

\section{Reagents}

Purchased ESR1 ligands included $17 \beta$-estradiol (E $\mathrm{E}_{2}$; Sigma), ICI 182780 (Tocris, Bristol, UK), tamoxifen (Sigma), Ralox (Tocris), and 4-hydroxytamoxifen (4OHT; Sigma). (R)6-(2-(N-(4-(2-(ethylamino)ethyl)benzyl)- $N$-ethylamino)4-methoxyphenyl)-5,6,7,8-tetrahydronaphthalen-2-ol dihydrochloride (RAD1901) was provided by Radius Pharmaceuticals (Waltham, MA, USA). BZA and GW7604 were synthesized as previously described (Wilson 1997,

Published by Bioscientifica Ltd. 
Miller et al. 2001). Ligands were dissolved in ethanol or DMSO.

\section{Cell culture}

Cell lines were authenticated by short tandem repeat (STRS) profiling performed by ATCC (Manassas, VA, USA) in 2013. Experiments were conducted using cell line passages 2-25. MCF7 and SKBR3 cell lines (ATCC) were maintained in DMEM/F12 or RPMI media (Invitrogen), respectively, supplemented with $8 \%(\mathrm{v} / \mathrm{v})$ fetal bovine serum (FBS; Gemini Bio-products, West Sacramento, CA, USA), nonessential amino acids (Invitrogen), and sodium pyruvate (Invitrogen). Unless otherwise indicated, cells were plated for experiments in media lacking phenol red and supplemented with 8\% charcoal stripped FBS (CFS; Gemini Bioproducts). Long-term estradiol deprivation (LTED) MCF7 cells were maintained and plated for experiments in phenol red free DMEM/F12 media supplemented with $8 \%$ CFS that had been charcoal stripped twice. Cells were treated with ESR1 ligands as indicated $48 \mathrm{~h}$ after plating and were harvested for immunoblot or real-time quantitative PCR analysis $24 \mathrm{~h}$ after treatment.

\section{Immunoblot analysis}

Protein expression was analyzed as described (Wittmann et al. 2007) using antibodies purchased from Sigma, A5441 ( $\beta$-actin) and Santa Cruz Biotechnology, sc-6259 (cytokeratin 18), sc-20680 (lamin A), sc-5546 ( $\alpha$-tubulin), and sc-8005 (ESR1).

\section{RNA isolation and real-time quantitative PCR}

RNA isolation and analysis was performed as described (Wardell et al. 2011). mRNA abundance was calculated using the $\Delta \Delta C_{\mathrm{T}}$ method (Wardell et al. 2011). Primer sequences are available on request.

\section{Proliferation assays}

Assays evaluating the effects of SERDs and SERMs on cell proliferation were performed as described (Wardell et al. 2013).

\section{Transfections}

Mammalian two-hybrid analysis of VP16-ESR1 with conformation-selective peptide probes was performed essentially as previously described (Wardell et al. 2012).

\section{In vivo studies}

All applicable international, national, and/or institutional guidelines for the care and use of animals were followed. All procedures performed in studies involving animals were in accordance with the ethical standards of the Duke University Institutional Animal Care and Use Committee.

Uterine wet weight analysis Ovariectomized (10 days prior) female C57Bl/6 mice (Charles River, Raleigh, NC, USA) were treated daily $(n=5)$ for 3 days with vehicle or estradiol benzoate (10 $\mu \mathrm{g} / \mathrm{kg}$ s.c.) as well as vehicle, Ralox (10 mg/kg s.c.), or RAD1901 (0.1-100 mg/kg s.c.). Ligands were dissolved in corn oil (Spectrum Chemicals, New Brunswick, NJ, USA). On day 4, mice were euthanized and tissues were retained for analysis. Uterine wet weight was calculated as a ratio of uterus weight on removal to body weight postmortem.

Xenograft tumor analysis Estrogen-stimulated MCF7 tumors were initiated in the axial mammary gland of 6-week-old estrogen-treated $(0.72 \mathrm{mg} / 60$ days pellet s.c.; Innovative Research of America, Sarasota, FL, USA) ovariectomized female NU/NU mice (in-house colony) by serial transfer and were measured as described (Wardell et al. 2013). For experiments with estrogen supplementation, at $\sim 0.1 \mathrm{~cm}^{3}$ tumor volume, mice were randomized $(n=9-10)$ to daily treatment with vehicle, RAD1901 $(20 \mathrm{mg} / \mathrm{kg})$, or tamoxifen $(20 \mathrm{mg} / \mathrm{kg})$. For experiments with estrogen withdrawal, at $\sim 0.1 \mathrm{~cm}^{3}$ tumor volume, the estrogen pellet was surgically removed, and mice were randomized $(n=6-10)$ to daily treatment with vehicle or RAD1901 (0.3-10 mg/kg). Treatments were formulated as above. Animal tissues were processed and analyzed as described (Wardell et al. 2013, 2015).

\section{Statistical analyses}

All statistical analyses were performed using GraphPad Prism 6 (San Diego, CA, USA) ( $P$ values are indicated in figure legends). Tumor growth was analyzed by exponential growth curve analysis and by two-way ANOVA of matched values followed by Bonferroni multiple comparisons to establish the significance between the groups at each day of treatment. Uterine weight, ESR1 expression in tumors and uteri, and cell proliferation (Fig. 5E) were compared by ANOVA followed by Bonferroni multiple comparisons. Comparisons of ESR1 target gene regulation were conducted by two-way ANOVA followed by Fisher's least significant difference test.

Published by Bioscientifica Ltd 


\section{Results}

\section{RAD1901 inhibits ESR1 activity in vitro and in vivo}

The ability of RAD1901 (Fig. 1A) to modulate the $\mathrm{E}_{2}$ dependent transcriptional activity of the human ESR1 and human ER beta (ESR2) was assessed in transiently transfected SKBR3 cells using a synthetic reporter gene. In this analysis it was determined that although RAD1901 effectively inhibits $\mathrm{E}_{2}$-dependent activation of an EREluciferase reporter by either isoform, it is a more potent inhibitor of ESR1 (100-fold; Fig. 1B). Similarly, it was demonstrated in MCF7 breast cancer cells that RAD1901 inhibited $E_{2}$-dependent $i$ ) induction of target gene transcription and ii) stimulation of cell proliferation with an efficacy and potency similar to that of the SERMs 4OHT and Ralox and the SERDs ICI 182780 (ICI, fulvestrant) and GW7604 (Fig. 1C and D). To assess the activity of RAD1901 in vivo, we conducted a xenograft tumor study in estrogen-treated immunocompromised mice using the well-characterized ESR1-dependent MCF7 cell model. In this study, it was observed that RAD1901 (20 mg/kg) inhibited $\mathrm{E}_{2}$-stimulated growth of the tumors with an efficacy similar to tamoxifen $(20 \mathrm{mg} / \mathrm{kg})$. Importantly, RAD1901 and tamoxifen were also shown to suppress the expression of the ESR1-target genes PGR and FHL1 to the same degree in treated tumors, a result that confirms target engagement (Fig. 1E and F).

\section{RAD1901 exhibits the pharmacological properties of a SERD}

Of late, there has been considerable interest in the development of compounds that not only function as ESR1 antagonists but also downregulate the expression of this receptor. It was of interest, therefore, that we demonstrated that ESR1 expression was significantly

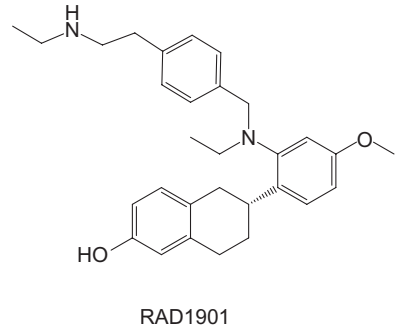

D

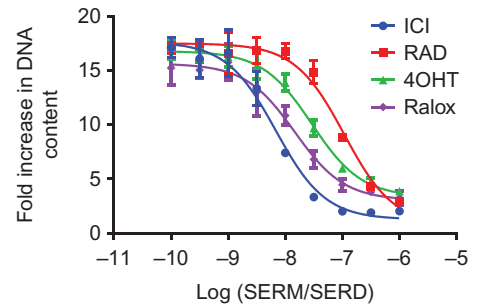

B

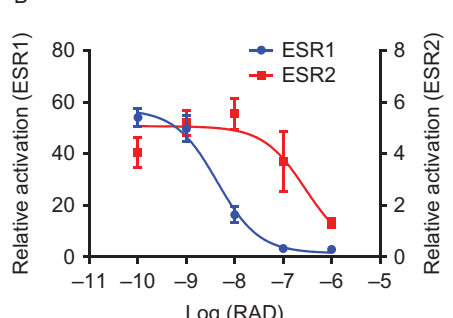

Log (RAD)

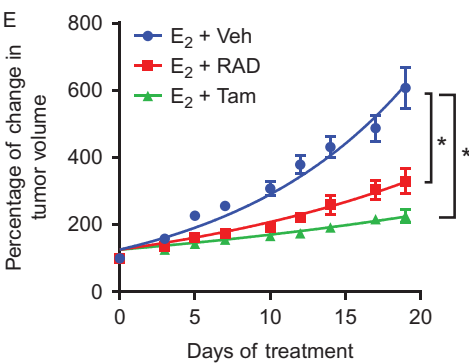

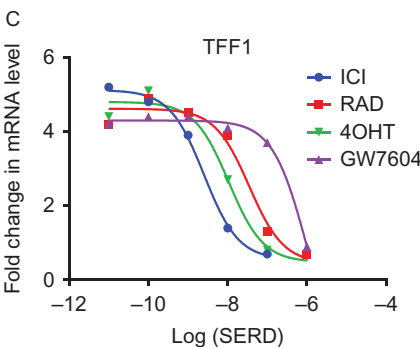

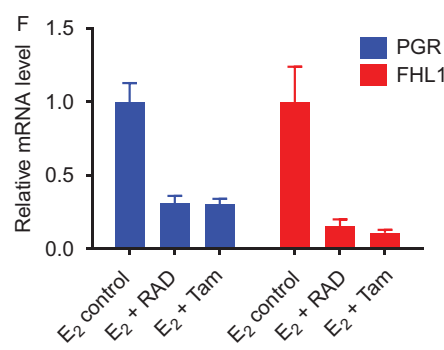

\section{Figure 1}

RAD1901 inhibits ESR1 activity in vitro and in vivo. (A) Chemical structure of SERM RAD1901. (B) SKBR3 cells were plated in phenol red free media supplemented with charcoal stripped FBS (CFS) $24 \mathrm{~h}$ prior to transfection with an ERE-luciferase reporter together with ESR1 or ESR2 expression vectors. Twenty-four hours after transfection, cells were treated with $E_{2}$ $(10 \mathrm{nM})$ together with RAD $\left(10^{-10}-10^{-6} \mathrm{M}\right)$ for $24 \mathrm{~h}$ prior to harvest and analysis of luciferase activity normalized to co-transfected $\beta$-galactosidase control. (B) MCF7 cells were plated in phenol red free media supplemented with CFS $48 \mathrm{~h}$ prior to treatment with $10^{-9} \mathrm{M} \mathrm{E}_{2}$ together with ICI 182780 (ICI), RAD1901 (RAD), GW7604, or 4-hydroxytamoxifen (4OHT) $\left(10^{-11}\right.$ $10^{-6} \mathrm{M}$ ) for $24 \mathrm{~h}$. mRNA levels of ESR1 target gene trefoil factor 1 (TFF1) were assessed using RT qPCR following RNA isolation. mRNA expression was normalized to the similarly detected 36B4 housekeeping gene, and expression levels are presented as fold change as compared to the vehicletreated control. (D) MCF7 cells were plated in phenol red free media supplemented with CFS $24 \mathrm{~h}$ prior to treatment and were treated with
$10^{-9} \mathrm{M} \mathrm{E}_{2}$ as well as with the indicated ligands $\left(10^{-11}-10^{-6} \mathrm{M}\right)$ on days 1,4 , and 6 of an 8-day proliferation assay. DNA content as assessed by fluorescence was measured as a surrogate for cell proliferation.

The relative increase in DNA fluorescence was calculated by normalizing to baseline values detected in a duplicate plate of cells that was harvested on day 1 prior to the initial treatment. Data are representative of at least three independent experiments. (E and F) MCF7 cell-derived tumors were implanted into ovariectomized estrogen-treated Nu/Nu mice. When tumor volume reached $\sim 0.1 \mathrm{~cm}^{3}$, animals $(n=9-10)$ were randomized to receive a daily treatment with vehicle, tamoxifen (Tam, $20 \mathrm{mg} / \mathrm{kg} \mathrm{s.c.)} \mathrm{or} \mathrm{RAD}$ (20 mg/kg s.C.). (E) Mean tumor volume \pm s.E.M. per day of treatment is presented. Significance (two-way ANOVA of matched values followed by Bonferroni's comparison) as compared to the vehicle control is indicated ( $\left.{ }^{*} P<0.0001\right)$. (F) Expression of ESR1 target genes in tumors was analyzed essentially as in (C). 

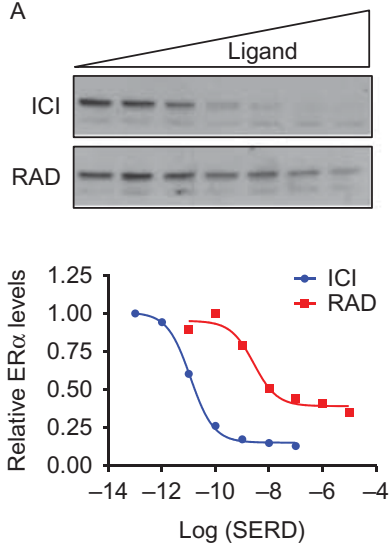

B
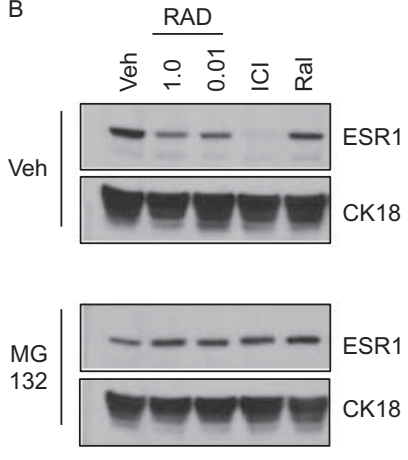

C

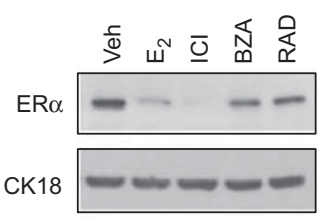

D

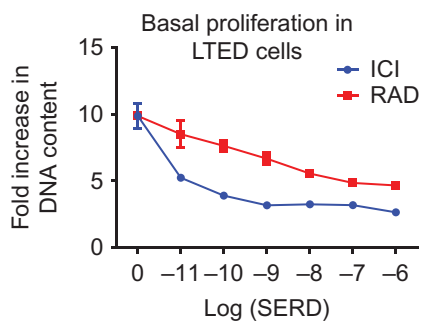

Figure 2

RAD1901 downregulates ESR1 expression through receptor degradation. (A) MCF7 cells were treated for $24 \mathrm{~h}$ with ICI $\left(10^{-13}-10^{-7} \mathrm{M}\right)$ or RAD $\left(10^{-11}-10^{-5} \mathrm{M}\right)$. Expression of ESR 1 and loading control cytokeratin 18 (CK18; Supplementary Figure 1A) in whole cell extracts was detected by immunoblot (top). ESR1 levels relative to CK18 were quantitated by densitometry using Adobe Photoshop (bottom). (B) MCF7 cells were plated as in Fig. 1B prior to $1 \mathrm{~h}$ pre-treatment with vehicle or MG132 $(10 \mu \mathrm{g} / \mathrm{ml})$, followed by $6 \mathrm{~h}$ of treatment with $10^{-7} \mathrm{M}$ vehicle, ICl, Ral, or RAD $\left(10^{-8}\right.$ or

downregulated in MCF7 cells treated with RAD1901. Whereas not as effective as the benchmark SERD fulvestrant, the downregulation of the ESR1 by RAD1901 is likely to be a significant contributor to its antagonist efficacy (Fig. 2A). Interestingly, RAD1901 had no effect on ESR1 mRNA expression (not shown). However, as observed in ICI-treated cells, the downregulation of ESR1 by RAD1901 was completely blocked by the pre-treatment of cells with the proteasome inhibitor MG132 (Fig. 2B). Similar results were obtained in the BT483 breast cancer cell line (Supplementary Figure 1B, C and D, see section on supplementary data given at the end of this article). For comparative purposes, we evaluated ESR1 expression levels in cells treated with tamoxifen, which stabilizes ESR1 expression, and SERDs BZA, GW7604, and ICI. These studies reveal that the degree of downregulation of ESR1 by RAD1901 was similar to that achieved by BZA under the same conditions (Fig. 2C). To assess the potential significance of this SERD activity, we evaluated the activity of RAD1901 in LTED MCF7 cells, an accepted model of aromatase resistance in which compounds with ER-antagonist activity alone, like tamoxifen, are minimally effective (not shown). In this assay, it was demonstrated that, like ICI and BZA, RAD1901 downregulated ESR1 expression and inhibited cell proliferation (Fig. 2D). Thus, considering its antagonist activity in several models of breast cancer and its ability to downregulate ESR1, it is appropriate to classify RAD1901 as a SERD.
$10^{-6} \mathrm{M}$ ). ESR1 expression was detected as in (A). (C and D) LTED MCF7 cells were plated in phenol red free media supplemented with FBS that was stripped of growth factors twice using charcoal. (C) After $48 \mathrm{~h}$, cells were treated for $24 \mathrm{~h}$ with $\mathrm{E}_{2}\left(10^{-7} \mathrm{M}\right)$ or SERDs $\left(10^{-6} \mathrm{M}\right)$, and ESR1 was analyzed as in (A). (D) LTED MCF7 cells were treated with ICI or RAD $\left(10^{-11}-10^{-6} \mathrm{M}\right)$ on days 1,4 , and 6 of an 8-day proliferation assay and analyzed as in Fig. 1.

\section{RAD1901 exhibits dose-dependent SERD activity in vivo}

The observation that RAD1901 exhibited the pharmacological properties of a SERD was unexpected given that it was i) identified in screens for compounds that manifest ESR1 agonist activity in the CNS and ii) evaluated in clinical trials as a potential treatment for the vasomotor symptoms (hot flashes) associated with menopause ( $\mathrm{O}^{\prime}$ Dea et al. 2010), an indication for which only estrogens have proven effective. However, the results of the clinical trials for hot flashes revealed that RAD1901 exhibited a complex pharmacology. At the lowest dose tested, it appeared to effectively suppress hot flashes but was ineffective at the higher doses tested (O'Dea et al. 2010). This inverted U-shaped pharmacology suggested to us that at low doses this compound may have favorable agonist activity, but at higher doses the ability of RAD1901 to induce ESR1 turnover dominates. Thus, we embarked on a series of studies to explore the functional consequences of the complex pharmacological activities of RAD1901 in vivo.

As a first step we evaluated the impact of RAD1901 on uterine wet weight in mice. For this study, increasing doses of RAD1901 were administered daily to ovariectomized C57Bl/6 mice receiving vehicle alone or $E_{2}(10 \mu \mathrm{g} / \mathrm{kg}$; a physiological replacement dose). A group of mice receiving $10 \mathrm{mg} / \mathrm{kg}$ Ralox was included for comparative purposes and for reference. When administered as a single agent, a statistically significant increase in uterine weight

Published by Bioscientifica Ltd 

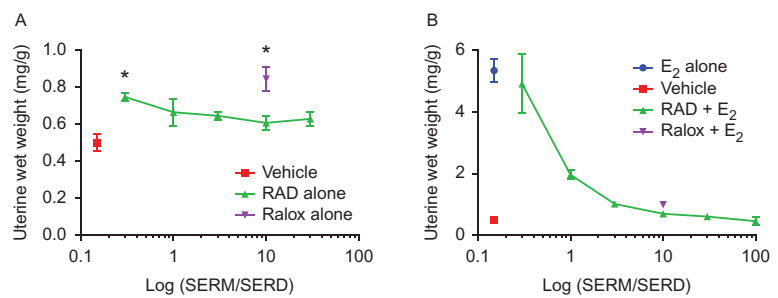

c

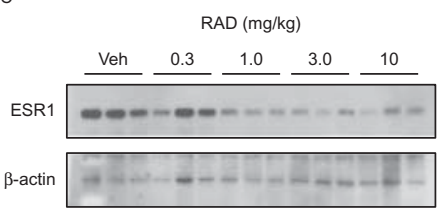

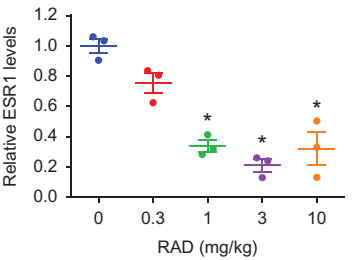

\section{Figure 3}

The in vivo pharmacology of RAD1901 is influenced by SERD activity. Ovariectomized C57BI/6 mice $(n=5)$ were treated daily for 3 days with (A) vehicle or (B) estradiol (10 $\mu \mathrm{g} / \mathrm{kg})$ together with vehicle, Ralox $(10 \mathrm{mg} / \mathrm{kg})$, or RAD $(0.3-100 \mathrm{mg} / \mathrm{kg})$. At euthanasia, body weight and uterine wet weight were measured prior to cryopreservation of the uterus. Significant changes in uterine weight observed in animals not receiving estrogen treatment $(A)$ were determined by ANOVA followed by Bonferroni's

was observed in those animals receiving the lowest dose of RAD1901 $(0.3 \mathrm{mg} / \mathrm{kg})$. At doses of $1 \mathrm{mg} / \mathrm{kg}$ and above the uterine wet weights of treated animals were indistinguishable from vehicle-treated animals (Fig. 3A). Further, RAD1901 administration was shown to inhibit $\mathrm{E}_{2}$-dependent increases in uterine wet weight (Fig. 3B), an activity that tracked with the dose-dependent downregulation of ESR1 expression (Fig. 3C). Notably, we did not observe any decrease in ESR1 expression in the animals treated with $0.3 \mathrm{mg} / \mathrm{kg}$, the dose where the agonist activity of RAD1901 in the uterine wet weight assay was observed (Fig. 3C). We conclude from these studies that, as observed in clinical trial for hot flashes, RAD1901 exhibits a complex biphasic pharmacology that manifests as antagonist activity at the higher doses.

\section{RAD1901 exhibits biphasic activity with respect to ESR1-dependent tumor growth}

As shown above, high dose RAD1901 (20 mg/kg) inhibited the growth of ESR1-dependent MCF7 cell-derived tumors in mice. However, the observation that uterine wet weight was increased in mice treated with doses of RAD1901 that were lower than that required to effect ESR1 turnover highlighted the need to examine whether a similar biphasic pharmacology was manifest in breast tumors. To further evaluate the pharmacology of RAD1901, we conducted a second xenograft tumor study in which MCF7 tumors were established under estrogen stimulation. When tumors reached $\sim 0.1 \mathrm{~cm}^{3}$ volume, estrogen was discontinued and animals were randomized to treatment with vehicle or $\operatorname{RAD} 1901(0.3,1,3$, or $10 \mathrm{mg} / \mathrm{kg})$. As observed in the uterine weight assay, RAD1901 exhibited a biphasic response, in that significant stimulation of tumor growth was observed in animals treated with 1 or $3 \mathrm{mg} / \mathrm{kg}$ RAD1901 that was not comparison to the vehicle control and are indicated $(* P<0.05)$. (C) ESR1 and $\beta$-actin expression in extracts made from pulverized uterine tissues were analyzed by immunoblot as in Fig. 1 (left). ESR1 expression relative to $\beta$-actin was quantitated as in Fig. 2 (right). Significant downregulation $\left({ }^{*} P<0.05\right)$ of ESR 1 was determined by ANOVA followed by Bonferroni's multiple comparison.

apparent at the higher dose (Fig. 4A). Although certainly less than that which was observed following $\mathrm{E}_{2}$ stimulation (Fig. 1E), the increased tumor volume in the 1 and $3 \mathrm{mg} / \mathrm{kg}$ groups is significant and of similar magnitude to that which we have reported previously for partial ESR1 agonists (Nelson et al. 2013). Interestingly, an evaluation of the final tumor size and uterine wet weight of these animals at sacrifice revealed that the pharmacology of RAD1901 is affected by both dose and tissue. Specifically, as reported above in a different strain of mice, stimulation of uterine weight was apparent in mice treated with only the $0.3 \mathrm{mg} / \mathrm{kg}$ dose of RAD1901, while tumor size was significantly increased in the 1 or $3 \mathrm{mg} / \mathrm{kg}$ RAD1901 groups (Fig. 4B). Whereas the expression of classical ESR1 target genes such as PGR (Fig. 4C) and others (MYBL1, HSPB8, FHL1, and CXCL12, not shown) was observed only in tumors treated with estrogen and was not observed in the tumors in the 1 or $3 \mathrm{mg} / \mathrm{kg}$ RAD1901 treatment groups (Fig. 4C), RAD1901 induction of AGR2 (Fig. 4D) and other SERM-regulated genes associated with tamoxifen resistance (KRT13, KRT15, AGR3, and RFTN1, not shown) was observed. Immunoblot analysis of tumor extracts revealed a dose-dependent downregulation of ESR1 by RAD1901 that reflected its actions as an inhibitor of tumor growth (Fig. 4E). Together these data highlight the complex pharmacology of RAD1901 and stress the importance of developing biomarkers that read on the partial agonist activity of the drug and that can be used for dose optimization in clinical studies.

\section{Elucidation of the mechanisms that distinguish RAD1901 from other SERDs}

We next sought to determine whether the dose-dependent agonist/antagonist activity of RAD1901 could be observed

Published by Bioscientifica Ltd 

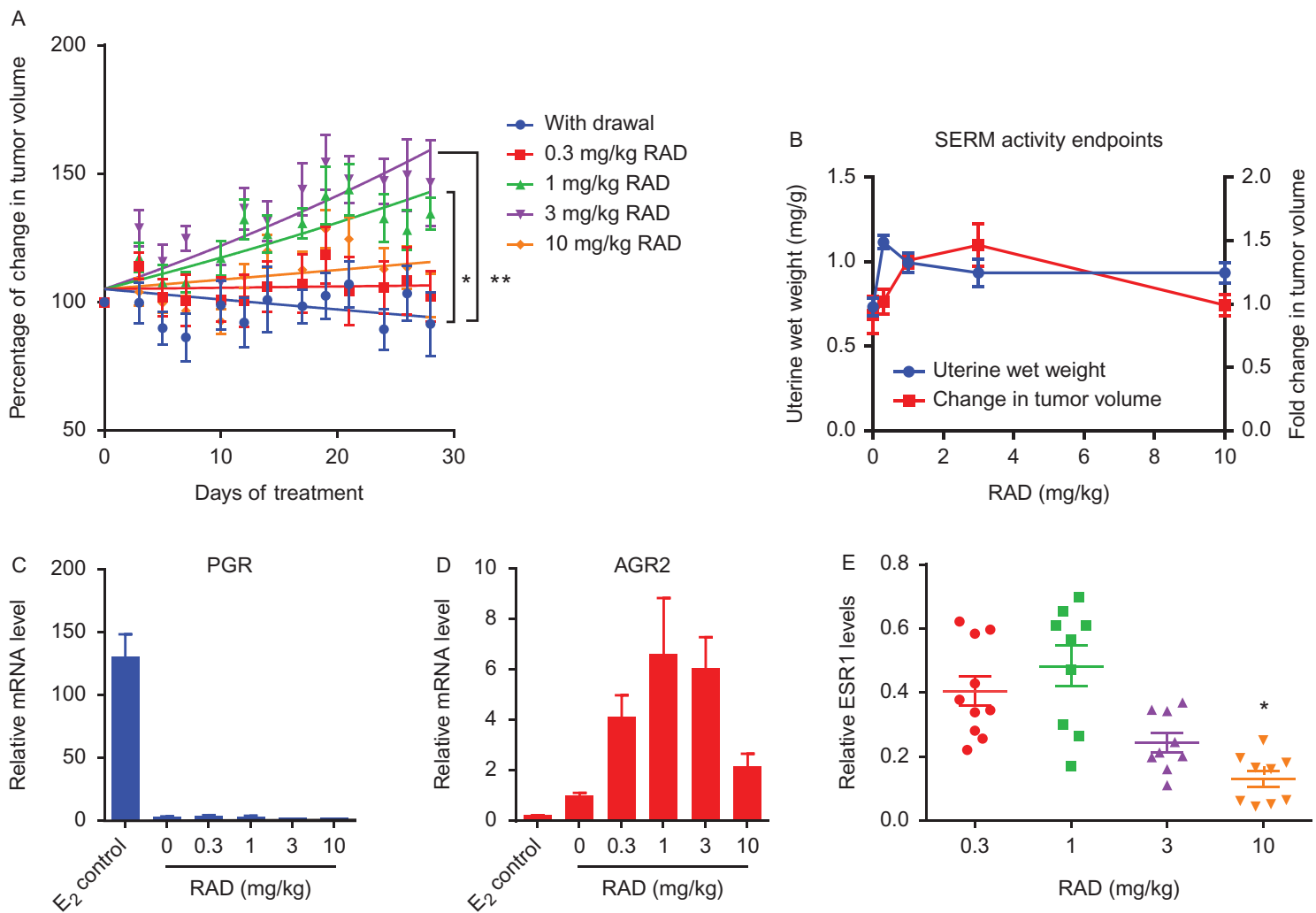

Figure 4

RAD1901 exhibits dose-dependent growth stimulation of MCF7 xenograft tumors. MCF7 xenograft tumors were initiated in ovariectomized female $\mathrm{Nu} / \mathrm{Nu}$ mice as in Fig. 1. Estrogen pellets were surgically removed when tumors reached $\sim 0.1 \mathrm{~cm}^{3}$ volume, and animals $(n=6-10)$ then received a daily treatment with vehicle or RAD (0.3-10 $\mathrm{mg} / \mathrm{kg}$ s.c.). Mean tumor volume \pm s.E.M. per day of treatment is presented. Significance as compared to the vehicle (two-way ANOVA of matched values followed by Bonferroni's comparison) is indicated ( $* P<0.05$ and $* * P<0.0005)$. (B) Uterine wet weight at sacrifice (measured as in Fig. 3 ) and \% change in tumor volume (as compared to size at randomization) calculated using the final measurement recorded for mice in $(A)$ are graphically presented. ( $C$ and D) Expression of ESR1 target genes in tumors was analyzed essentially as in Fig. 1. Estrogen only samples from Fig. 1D were included for comparison. (E) ESR1 levels in tumor tissues were analyzed as in Fig. 3 and were normalized to similarly detected Lamin-A. Significant downregulation ( $\left.{ }^{*} P<0.05\right)$ of ESR1 was determined by ANOVA followed by Bonferroni's comparison. at the level of target gene regulation. Previously, we reported on the identification of specific sets of ESR1 target genes in which the expression was differentially regulated by estrogens and SERMs and that could be used to distinguish between different SERMs and SERDs (Wardell et al. 2012). A subset of these genes, those regulated by i) agonists (with no or minimal response to SERMs), ii) SERMs (with no or little response to $E_{2}$ ), or iii) either agonists or SERMs, were selected to profile RAD1901 activity (Wardell et al. 2012). Reflecting the pharmacology observed in vivo, the expression pattern of these target genes exhibited a biphasic response to RAD1901 with agonist activity observed at lower doses and more complete antagonist activity apparent at higher doses (Fig. 5A, B, C and Supplementary Figure 2, see section on supplementary data given at the end of this article). These data confirm the unique pharmacology of RAD1901 and suggest that this drug may function by a mechanism that is distinct from other ESR1 downregulators.

It is now generally accepted that, notwithstanding differences in pharmaceutical properties, the pharmacology of ESR1 ligands reflects their influence on the overall structure of the receptor and on the impact that this has on coregulator recruitment. Given the distinct pharmacology exhibited by RAD1901, we hypothesized that it may enable ESR1 to adopt a unique conformation. To address this, we took advantage of a conformational profiling tool we reported previously to interrogate the ESR1-RAD1901 complex (Norris et al. 1999, Wardell et al. 2013). In this assay a modified two-hybrid assay is used to

Published by Bioscientifica Ltd. 


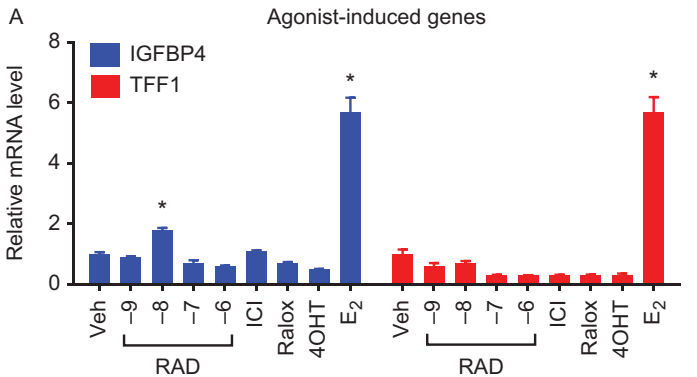

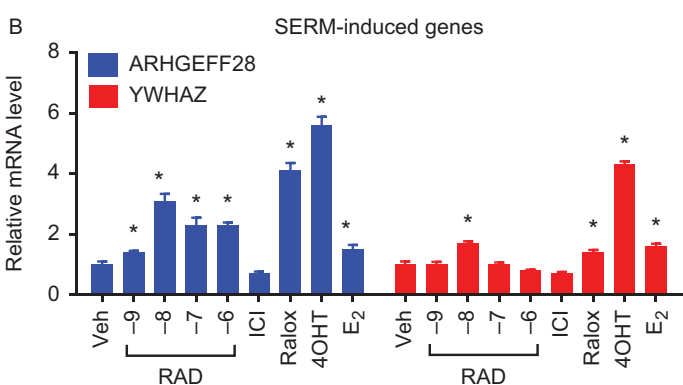
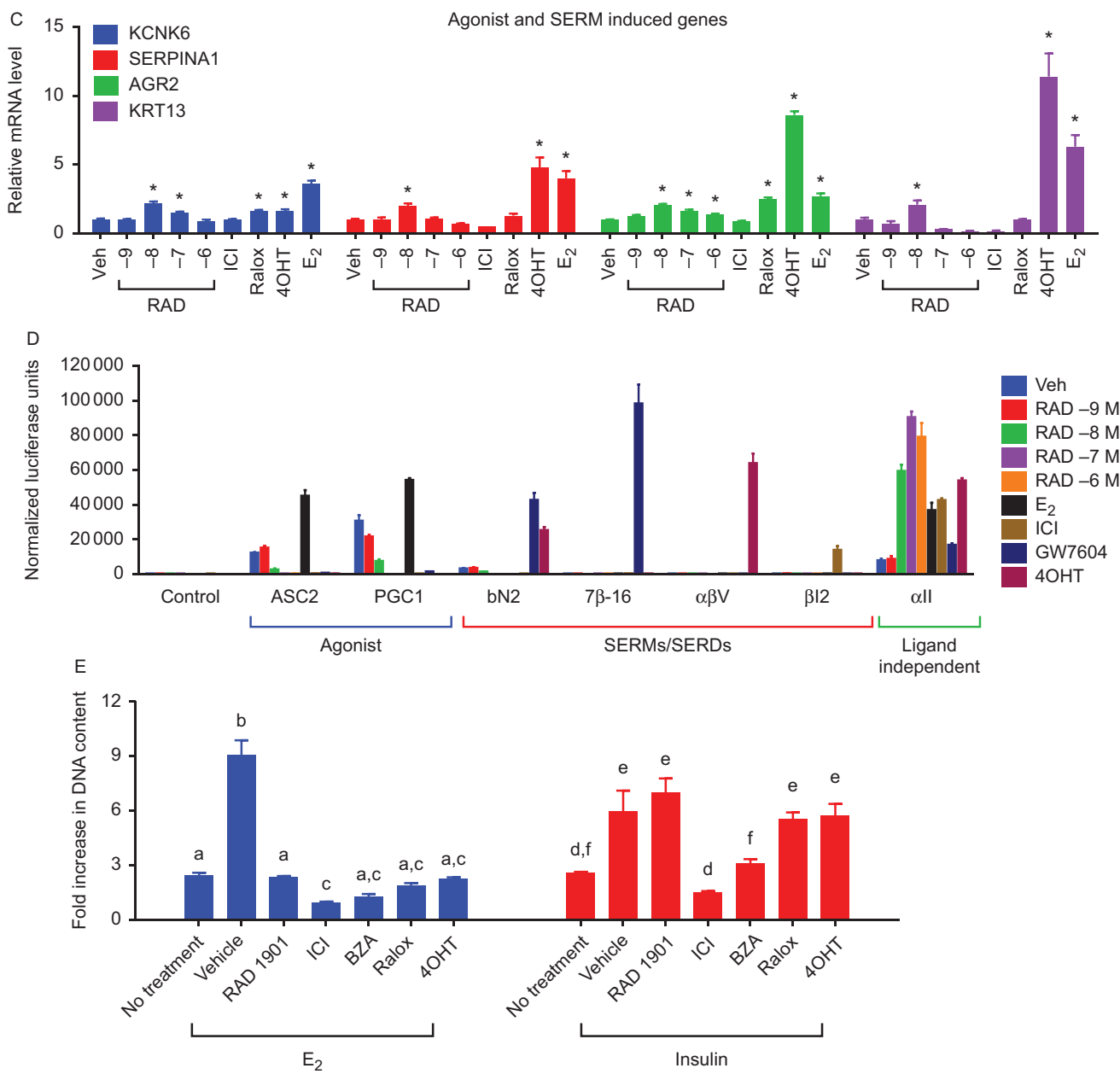

Figure 5

RAD1901 exerts biphasic agonist/antagonist activity on ESR1 in a dose dependent manner. (A) MCF7 cells were treated for $24 \mathrm{~h}$ with $10^{-7} \mathrm{M}$ vehicle (Veh), ICl, 4OHT, raloxifene (Ralox), $\mathrm{E}_{2}\left(10^{-9} \mathrm{M}\right)$, or RAD1901 (10 ${ }^{-9}$ $\left.10^{-6} \mathrm{M}\right)$. The expression of ESR 1 target genes responsive to $(A)$ agonists, (B) primarily SERMs, or (C) SERMs and agonists was analyzed as in Fig. 1. Relative changes of these and additional target genes designed to evaluate the dose-dependent response to RAD1901 are presented in Supplementary Figure 2 . Significant target gene regulation $\left({ }^{*} P<0.05\right)$ as compared to the vehicle control was detected by two-way ANOVA followed by Fisher's least significant difference (LSD) test. (D) Interaction between ESR1 and conformation-specific peptides in a mammalian two-hybrid system. Triplicate wells of SKBR3 cells were transfected with plasmids expressing ESR1 fused to VP16 together with Gal4DBD alone (control) or Gal4DBD fused to ESR 1 interacting peptides noted on the horizontal axis. Cells were then treated with the indicated ESR 1 ligands $\left(10^{-7} \mathrm{M}\right.$ unless otherwise indicated). Interaction of ESR1 with the Gal4DBD peptide constructs was detected through the activation of a Gal4-responsive luciferase reporter construct and was normalized to detected $\beta$-galactosidase activity expressed in a constitutive manner using a second vector. Normalized response is expressed as fold increase over the detected level of interaction between Gal4DBD alone and ESR1-VP16 in the absence of ligand (Veh). E) The effect of SERMs and SERDs $\left(10^{-6} \mathrm{M}\right)$ on the proliferation of MCF7 cells in response to $E_{2}\left(10^{-9} \mathrm{M}\right)$ or insulin $\left(2 \times 10^{-9} \mathrm{M}\right)$ was evaluated as in Fig. 1. Statistically similar treatments (two-way ANOVA followed by Bonferroni's multiple comparison) are indicated by letters. 
assess the binding of a series of short peptides that survey the protein-protein interaction surfaces on ESR1 that are presented when occupied by different ligands. As shown in Fig. 5D, the interaction profile observed in the presence of RAD1901 is completely distinct from any other known ligand. The ability of RAD1901 to disengage the classical coregulator-binding surface (AF2) as indicated by the decreased interaction with the peptides that report on 'agonist activity' (ASC1 and PGC1) is notable. No interaction is observed with peptides that report on the ESR1 structures adopted upon binding tamoxifen $(\alpha \beta \mathrm{V})$ or ICI ( $\beta$ I2). There is, however, a significant interaction of $\alpha$ II in the presence of RAD1901, a peptide that reports on a cryptic protein-protein interaction surface in the hinge region of the receptor - the significance of which remains to be determined. It is concluded, therefore, that RAD1901 enables ESR1 to assume a unique conformation that is distinct from that apparent upon binding agonists, the SERM tamoxifen, and other known SERDs (Fig. 5D).

In addition to classical agonists, ESR1 transcriptional activity can also be induced by treating cells with growth factors such as EGF, IGF1, or insulin. Whereas the mechanisms underlying this 'ligand-independent' activity are likely to be complex, it is widely held that this alternate pathway of activation contributes to the resistance to endocrine therapy in breast cancer (Johnston et al. 2003). Therefore, as a final step in our evaluation of the comparative pharmacology of RAD1901, we evaluated its ability to suppress the growth factor dependent activation of ESR1 (Wardell et al. 2013). In this assay, it was determined that although all of the SERMs and SERDs tested inhibited $\mathrm{E}_{2}$-stimulated proliferation, BZA and ICI efficiently inhibited insulin-stimulated proliferation, whereas 4OHT, Ralox, and RAD1901 were without effect (Fig. 5E). These results again confirm the unique mechanism of action of RAD1901 and also highlight that this drug may need to be delivered in combination with another drug that inhibits ligand-independent activation of ESR1 to achieve maximal clinical response.

\section{Discussion}

In this study it was demonstrated that the ESR1 modulator RAD1901 exhibits a complex pharmacology, functioning as a weak partial agonist activity at lower doses of the compound and as an antagonist at higher doses. Whereas the molecular basis for the partial agonist activity remains elusive, it was observed that the binding of RAD1901 enabled the presentation of protein-protein interaction surfaces within the hinge region (flexible structure linking the DNA and hormone-binding domains) of ESR1 that we have previously shown to be important for transcriptional activity. The identification of the coregulators that interact with these surfaces on ESR1 will enable a definition of their importance in RAD1901 pharmacology. Importantly, however, it was also observed that, as the occupancy of the receptor increases, it is targeted for degradation and that this results in a quantitative inhibition of ESR1 signaling. Interestingly, this pharmacological profile resembles that of a classical agonist, such as $E_{2}$, in which the agonist signal is terminated by proteasome-dependent receptor degradation. It has already been established that the ability to induce transcriptional activation and receptor turnover is an integrally linked activity of ESR1 agonists. The findings of our study are consistent with the model that the pharmacological actions of RAD1901 represent an uncoupling of ESR1-dependent transcriptional activation from degradation. Genetic experiments are underway to define the components of the processes required for ESR1 turnover by RAD1901 and other ligands. The results of these studies should be informative with respect to differences in the mechanisms that regulate ESR1 stability in breast cancer and should help patient stratification of breast cancer patients in which ESR1 is the primary target. Regardless, we conclude that at appropriate doses the SERD activity manifest by RAD1901 may result in useful clinical activity in breast cancer in some settings.

RAD1901 is unique among SERMs and SERDs in that it readily crosses the blood-brain barrier. Thus, not surprisingly there was significant interest in the results of clinical studies that evaluated its activity as a treatment for vasomotor instability (hot flashes; O'Dea et al. 2010). Unfortunately, the results of the studies completed thus far were equivocal. Patients on lower doses of the drug clearly demonstrated benefits, whereas exposure to higher doses was ineffective or associated with a worsening of response. We considered potential mechanistic explanations for this pharmacology and herein present data suggesting that RAD1901 manifests partial agonist activity when assessed on gene transcription, in assays of uterine function, and in cellular and animal models of breast cancer at doses in which ESR1 degradation is not apparent. However, at doses of RAD1901 in which ESR1 degradation occurs, we note that the drug quantitatively inhibits the activity of this axis. Studies examining RAD1901 as a treatment for hot flashes at doses lower than that originally evaluated appear justified, although it remains to be determined if such protocols would have to include a

Published by Bioscientifica Ltd 
progestin to mitigate potential liabilities in the uterus that may be manifest at such doses.

The establishment of the relationship between the conformation of ESR1/ligand complexes, coregulator recruitment, and phenotypic response was an important advance in efforts to discover new molecules with unique pharmacological activities (Wardell et al. 2014). Most of these efforts have focused on the identification of SERMs for use in the prevention and treatment of osteoporosis, for breast cancer prevention in high risk patients, and for dyspareunia. Of late, however, there has been significant interest in developing SERDs for use in patients with advanced breast cancer. The first SERD approved, ICI, was identified in an empirical manner. However, a series of new, orally bioavailable drugs that exhibit substantial SERD activity and that all preclinical evidence suggests should have utility in the treatment of advanced disease are emerging from specific mechanism-based screens (Mayer et al. 2013). The most clinically advanced of these molecules, ARN810 (GDC-0810), induces ESR1 turnover by a mechanism distinct from ICI and thus may have advantages as an intervention in certain patient populations (Lai et al. 2015). BZA, a SERM, was also determined to induce substantial ESR1 turnover although the mechanism by which this occurs is elusive (LewisWambi et al. 2011, Wardell et al. 2013). The latter molecule is approved in several countries for the treatment and prevention of osteoporosis, and near term clinical studies to evaluate its efficacy in breast cancer are anticipated (Komm et al. 2005). With the number of options available, the challenge, therefore, with a new molecule like RAD1901 is to identify the clinical niche where it might have particular utility in the clinic. In cellular and animal models of breast cancer, this drug does not suppress ESR1 activity as well as other SERMs and SERDs, and its complex pharmacology may be problematic in classical dose escalation studies in which tumor growth/progression may occur at lower doses. Further, it has been determined that RAD1901 does not inhibit growth factor-mediated induction of ESR1 transcriptional activity, a significant liability in our estimation. However, the demonstrated ability of RAD1901 to cross the blood-brain barrier, a shortcoming of other SERMs and SERDs, is a significant positive attribute that has led us to propose to the innovators that this drug may have particular utility in the treatment of ESR1 positive breast cancer brain metastasis (BCBM).

Advancements in systemic breast cancer therapies have led to an unanticipated increase in the incidence of BCBM (Frisk et al. 2012). This is likely a consequence of the fact that metastasis to the brain is a very slow processes in ESR1-positive breast cancers and, thus, more apparent in long-term survivors. However, few treatments can access the brain, one of the three most frequent sites of metastasis for breast cancer, leaving targeted or whole brain radiation as the standard of care for patients diagnosed with BCBM (Freedman \& Anders 2012). Importantly, in the majority of BCBM occurring in patients diagnosed with ESR1-positive breast tumors, ESR1 expression is retained, and the high expression of aromatase in the brain suggests that the disruption of ESR1 signaling may be beneficial in this setting (Duchnowska et al. 2012). Indeed, anecdotal evidence would support a role for ESR1 as a therapeutic target in BCBM (Pors et al. 1991, Salvati et al. 1993, Madhup et al. 2006). While several SERDs are currently in development for the treatment of breast cancers that have progressed during endocrine intervention, and their approval is anticipated to be beneficial in managing peripheral disease, the ability of these drugs to cross the blood-brain barrier is currently unknown. However, RAD1901 is not only brain penetrative; it was shown to inhibit ESR1 signaling in the vasomotor symptom trial upon dose escalation ( $\mathrm{O}^{\prime}$ Dea et al. 2010). Further, 18-F fluoroestradiol-positron emission tomography (FES-PET) imaging recently demonstrated reduced $\mathrm{E}_{2}$ binding within the brain of healthy volunteers administered a relatively high dose of RAD1901 (Hattersley et al. 2014). Although these analyses do not measure ESR1 levels, as originally claimed, they do confirm substantial target engagement of ESR1 in the brain. Because patients diagnosed with BCBM are likely to have already progressed following endocrine therapies, the utility of RAD1901 in this setting may be dictated by the pharmacology exhibited by RAD1901 in breast tumors resistant to tamoxifen and/or AIs. Regardless, in the absence of a SERD with a less complicated pharmacological profile, RAD1901 may be beneficial in the BCBM setting when combined with additional therapeutic(s) that will ensure the inhibition of the growth of peripheral metastases.

\section{Supplementary data}

This is linked to the online version of the paper at http://dx.doi.org/10.1530/ ERC-15-0287.

\section{Declaration of interest}

D P McDonnell has previously served as a scientific advisory board member for Radius Pharmaceuticals. D P McDonnell, S E Wardell, and E R Nelson have applied for a patent for the use of RAD1901 for the treatment of breast cancer brain metastases.

Published by Bioscientifica Ltd. 


\section{Funding}

Supporting funding for all of the authors was provided by the National Institutes of Health (grant number R37DK048807) and a research grant from Radius Pharmaceuticals.

\section{References}

Bentrem DJ, Dardes RC, Liu H, Maccgregor-Schafer J, Zapf JW \& Jordan VC 2001 Molecular mechanism of action at estrogen receptor $\alpha$ of a new clinically relevant antiestrogen (GW7604) related to tamoxifen. Endocrinology 142 838-846.

Carter D 2014 New global survey shows an increasing cancer burden. American Journal of Nursing 11417.

Chia S, Gradishar W, Mauriac L, Bines J, Amant F, Federico M, Fein L, Romieu G, Buzdar A, Robertson J et al. 2008 Double-blind, randomized placebo controlled trial of fulvestrant compared with exemestane after prior nonsteroidal aromatase inhibitor therapy in postmenopausal women with hormone receptor-positive, advanced breast cancer: results from EFECT. Journal of Clinical Oncology 26 1664-1670. (doi:10.1200/JCO.2007.13.5822)

Connor CE, Norris JD, Broadwater G, Willson TM, Gottardis MM, Dewhirst MW \& McDonnell DP 2001 Circumventing tamoxifen resistance in breast cancers using antiestrogens that induce unique conformational changes in the estrogen receptor. Cancer Research 61 2917-2922.

Dallenbach-Hellweg G, Schmidt D, Hellberg P, Bourne T, Kreuzwieser E, Doren M, Rydh W, Rudenstam G \& Granberg S 2000 The endometrium in breast cancer patients on tamoxifen. Archives of Gynecology and Obstetrics 263 170-177. (doi:10.1007/s004040050276)

Dardes RC, O'Regan RM, Gajdos C, Robinson SP, Bentrem D, De Los Reyes A \& Jordan VC 2002 Effects of a new clinically relevant antiestrogen (GW5638) related to tamoxifen on breast and endometrial cancer growth in vivo. Clinical Cancer Research 8 1995-2001.

Duchnowska R, Dziadziuszko R, Trojanowski T, Mandat T, Och W, Czartoryska-Arlukowicz B, Radecka B, Olszewski W, Szubstarski F, Kozlowski $\mathrm{W}$ et al. 2012 Conversion of epidermal growth factor receptor 2 and hormone receptor expression in breast cancer metastases to the brain. Breast Cancer Research 14 R119. (doi:10.1186/bcr3244)

Freedman RA \& Anders CK 2012 Treatment of breast cancer brain metastases. Current Breast Cancer Reports 4 1-9. (doi:10.1007/s12609011-0061-5)

Frisk G, Svensson T, Backlund LM, Lidbrink E, Blomqvist P \& Smedby KE 2012 Incidence and time trends of brain metastases admissions among breast cancer patients in Sweden. British Journal of Cancer 106 1850-1853. (doi:10.1038/bjc.2012.163)

Hattersley G, Paquin D, Ho S, Merchenthaler I, Ogasawara A, Nakagawa M, Ogura H \& Lyttle CR 2007 RAD-1901, a novel SERM, has efficacy in an animal model of vasomotor symptoms. In 89th Annual Meeting of the Endocrine Society, Toronto, Ontario, Canada. Abstract P1-415.

Hattersley G, David F, Harris A, Clarkin M, Banks K, Williams G, Glaudemans A, Doorduin J, Koole M, de Vries E et al. 2014 RAD1901, a novel tissue-selective estrogen receptor degrader (SERD) demonstrates estrogen receptor engagement in a phase 1 clinical study. In San Antonio Breast Cancer Meeting, San Antonio, Texas, USA. Abstract OT2-1-10.

Jeselsohn R, Yelensky R, Buchwalter G, Frampton G, Meric-Bernstam F, Gonzalez-Angulo AM, Ferrer-Lozano J, Perez-Fidalgo JA, Cristofanilli M, Gomez H et al. 2014 Emergence of constitutively active estrogen receptor- $\alpha$ mutations in pretreated advanced estrogen receptor-positive breast cancer. Clinical Cancer Research 20 1757-1767. (doi:10.1158/ 1078-0432.CCR-13-2332)

Johnston SR, Head J, Pancholi S, Detre S, Martin LA, Smith IE \& Dowsett M 2003 Integration of signal transduction inhibitors with endocrine therapy: an approach to overcoming hormone resistance in breast cancer. Clinical Cancer Research 9 524S-532S.
Komm BS \& Chines AA 2012 An update on selective estrogen receptor modulators for the prevention and treatment of osteoporosis. Maturitas 71 221-226. (doi:10.1016/j.maturitas.2011.11.018)

Komm BS, Kharode YP, Bodine PV, Harris HA, Miller CP \& Lyttle CR 2005 Bazedoxifene acetate: a selective estrogen receptor modulator with improved selectivity. Endocrinology 146 3999-4008. (doi:10.1210/en. 2005-0030)

Lai A, Kahraman M, Govek S, Nagasawa J, Bonnefous C, Julien J, Douglas K, Sensintaffar J, Lu N, Lee KJ et al. 2015 Identification of GDC-0810 (ARN810 ), an orally bioavailable selective estrogen receptor degrader (SERD) that demonstrates robust activity in tamoxifen-resistant breast cancer xenografts. Journal of Medicinal Chemistry 58 4888-4904. (doi:10.1021/ acs.jmedchem.5b00054)

Leo AD, Jerusalem G, Petruzelka L, Torres R, Bondarenko I, Khasanov R, Verhoeven D, Pedrini J, Lichinitser M, Pendergrass K et al. 2009 CONFIRM: a phase III, randomized, parallel-group trial comparing fulvestrant $250 \mathrm{mg}$ vs fulvestrant $500 \mathrm{mg}$ in postmenopausal women with estrogen receptor-positive advanced breast cancer. Cancer Research 69 Abstract no. 25. (doi:10.1158/0008-5472.SABCS-09-25)

Lewis-Wambi JS, Kim H, Curpan R, Grigg R, Sarker MA \& Jordan VC 2011 The selective estrogen receptor modulator bazedoxifene inhibits hormone-independent breast cancer cell growth and down-regulates estrogen receptor $\alpha$ and cyclin D1. Molecular Pharmacology 80 610-620. (doi:10.1124/mol.111.072249)

Lindahl B, Andolf E, Ingvar C, Ranstam J \& Willen R 2008 Adjuvant tamoxifen in breast cancer patients affects the endometrium by time, an effect remaining years after end of treatment and results in an increased frequency of endometrial carcinoma. Anticancer Research $\mathbf{2 8}$ 1259-1262.

Madhup R, Kirti S, Bhatt ML, Srivastava PK, Srivastava M \& Kumar S 2006 Letrozole for brain and scalp metastases from breast cancer - a case report. Breast 15 440-442. (doi:10.1016/j.breast.2005.07.006)

Mayer I, Bardia A, Dickler M, Manning H, Mahmood U, Ulaner G, Hager J, Rix P, Zack N, Maneval E et al. 2013 Abstract OT3-2-07: Phase I study of ARN-810, a novel selective estrogen receptor degrader, in postmenopausal women with locally advanced or metastatic estrogen receptor positive breast cancer. Cancer Research $\mathbf{7 3}$ Abstract no. OT3-2-07. (doi:10.1158/0008-5472.SABCS13-OT3-2-07)

Miller CP, Collini MD, Tran BD, Harris HA, Kharode YP, Marzolf JT, Moran RA, Henderson RA, Bender RH, Unwalla RJ et al. 2001 Design, synthesis, and preclinical characterization of novel, highly selective indole estrogens. Journal of Medicinal Chemistry 44 1654-1657. (doi:10.1021/jm010086m)

Nelson ER, Wardell SE, Jasper JS, Park S, Suchindran S, Howe MK, Carver NJ, Pillai RV, Sullivan PM, Sondhi V et al. 2013 27-Hydroxycholesterol links hypercholesterolemia and breast cancer pathophysiology. Science $\mathbf{3 4 2}$ 1094-1098. (doi:10.1126/science.1241908)

Norris JD, Paige LA, Christensen DJ, Chang CY, Huacani MR, Fan D, Hamilton PT, Fowlkes DM \& McDonnell DP 1999 Peptide antagonists of the human estrogen receptor. Science 285 744-746. (doi:10.1126/ science.285.5428.744)

O'Dea L, McCarthy D, Guerriero J, Miller C, Hattersley G \& Lyttle CR 2010 RAD1901, a novel selective estrogen receptor modulator (SERM), demonstrates evidence of efficacy on postmenopausal hot flashes in an early phase human study. In 92nd Annual Meeting of the Endocrine Society, San Diego, California, USA. Abstract P1-726.

Perey L, Paridaens R, Hawle H, Zaman K, Nole F, Wildiers H, Fiche M, Dietrich D, Clement P, Koberle D et al. 2007 Clinical benefit of fulvestrant in postmenopausal women with advanced breast cancer and primary or acquired resistance to aromatase inhibitors: final results of phase II Swiss Group for Clinical Cancer Research Trial (SAKK 21/00). Annals of Oncology 18 64-69. (doi:10.1093/annonc/mdl341)

Pors H, von Eyben FE, Sorensen OS \& Larsen M 1991 Longterm remission of multiple brain metastases with tamoxifen. Journal of Neuro-Oncology $\mathbf{1 0}$ 173-177. (doi:10.1007/BF00146879) 
Riggins RB, Schrecengost RS, Guerrero MS \& Bouton AH 2007 Pathways to tamoxifen resistance. Cancer Letters 256 1-24. (doi:10.1016/j.canlet. 2007.03.016)

Robertson J, Nicholson R, Bundred N, Anderson E, Rayter Z, Dowsett M, Fox J, Gee J, Webster A, Wakeling A et al. 2001 Comparison of the shortterm biological effects of $7 \alpha$-[9-(4,4,5,5,5-pentafluoropentylsulfinyl)nonyl]estra-1,3,5, (10)-triene-3,17 $\beta$-diol (Faslodex) versus tamoxifen in postmenopausal women with primary breast cancer. Cancer Research 61 6739-6746.

Robertson J, Llombart-Cussac A, Feltl D, Dewar J, Jasiówka M, Hewson N, Rukazenkov Y \& Ellis M 2014 Fulvestrant $500 \mathrm{mg}$ versus anastrozole as first-line treatment for advanced breast cancer: overall survival from the phase II 'FIRST' study. In San Antonio Breast Cancer Symposium. San Antionio, Texas, USA. Abstract S6-04.

Robinson DR, Wu YM, Vats P, Su F, Lonigro RJ, Cao X, Kalyana-Sundaram S, Wang R, Ning Y, Hodges L et al. 2013 Activating ESR1 mutations in hormone-resistant metastatic breast cancer. Nature Genetics $\mathbf{4 5}$ 1446-1451. (doi:10.1038/ng.2823)

Salvati M, Cervoni L, Innocenzi G \& Bardella L 1993 Prolonged stabilization of multiple and single brain metastases from breast cancer with tamoxifen. Report of three cases. Tumori 79 359-362.

Toy W, Shen Y, Won H, Green B, Sakr RA, Will M, Li Z, Gala K, Fanning S, King TA et al. 2013 ESR1 ligand-binding domain mutations in hormone-resistant breast cancer. Nature Genetics 45 1439-1445. (doi:10.1038/ng.2822)

Wardell SE, Marks JR \& McDonnell DP 2011 The turnover of estrogen receptor $\alpha$ by the selective estrogen receptor degrader (SERD) fulvestrant is a saturable process that is not required for antagonist efficacy. Biochemical Pharmacology 82 122-130. (doi:10.1016/j.bcp.2011. 03.031)
Wardell SE, Kazmin D \& McDonnell DP 2012 Research resource: Transcriptional profiling in a cellular model of breast cancer reveals functional and mechanistic differences between clinically relevant SERM and between SERM/estrogen complexes. Molecular Endocrinology 26 1235-1248. (doi:10.1210/me.2012-1031)

Wardell SE, Nelson ER, Chao CA \& McDonnell DP 2013 Bazedoxifene exhibits antiestrogenic activity in animal models of tamoxifenresistant breast cancer: implications for treatment of advanced disease. Clinical Cancer Research 19 2420-2431. (doi:10.1158/1078-0432.CCR12-3771)

Wardell SE, Nelson ER \& McDonnell DP 2014 From empirical to mechanism-based discovery of clinically useful selective estrogen receptor modulators (SERMs). Steroids 90 30-38. (doi:10.1016/j. steroids.2014.07.013)

Wardell SE, Ellis MJ, Alley HM, Eisele K, VanArsdale T, Dann SG, Arndt KT, Primeau T, Griffin E, Shao J et al. 2015 Efficacy of SERD/SERM hybrid-CDK4/6 inhibitor combinations in models of endocrine therapy resistant breast cancer. Clinical Cancer Research [in press]. (doi:10.1158/1078-0432.CCR-15-0360)

Willson TM, Norris JD, Wagner BL, Asplin I, Baer P, Brown HR, Jones SA, Henke B, Sauls H, Wolfe S et al. 1997 Dissection of the molecular mechanism of action of GW5638, a novel estrogen receptor ligand, provides insights into the role of ER in bone. Endocrinology $\mathbf{1 3 8}$ 3901-3911.

Wilson T 1997 A method of preventing or treating estrogen-dependent diseases and disorders. US patent \# US 5681835 A. United States: Glaxo Wellcome, Inc.

Wittmann BM, Sherk A \& McDonnell DP 2007 Definition of functionally important mechanistic differences among selective estrogen receptor down-regulators. Cancer Research 67 9549-9560. (doi:10.1158/00085472.CAN-07-1590)

Received in final form 6 July 2015

Accepted 8 July 2015
(C) 2015 Society for Endocrinology Printed in Great Britain 